\title{
BMJ Open What are the most effective behaviour change techniques to promote physical activity and/or reduce sedentary behaviour in inactive adults? A systematic review protocol
}

\author{
Neil Howlett, ${ }^{1}$ Daksha Trivedi, ${ }^{2}$ Nicholas A Troop, ${ }^{1}$ Angel Marie Chater ${ }^{3}$
}

To cite: Howlett N, Trivedi D, Troop NA, et al. What are the most effective behaviour change techniques to promote physical activity and/ or reduce sedentary

behaviour in inactive adults? A systematic review protocol. BMJ Open 2015;5: 0008573. doi:10.1136/bmjopen-2015008573

- Prepublication history for this paper is available online. To view these files please visit the journal online (http://dx.doi.org/10.1136/ bmjopen-2015-008573)

Received 22 April 2015 Revised 1 July 2015

Accepted 2 July 2015

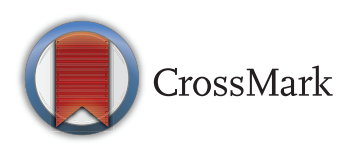

${ }^{1}$ Department of Psychology, University of Hertfordshire, Hatfield, Hertfordshire, UK ${ }^{2}$ Centre for Research in Primary and Community Care, University of Hertfordshire, Hatfield, Hertfordshire, UK

${ }^{3}$ Research Department of Practice and Policy, Centre for Behavioural Medicine, UCL School of Pharmacy, University College London, London, UK

Correspondence to Dr Angel Chater; a.chater@ucl.ac.uk

\section{ABSTRACT}

Introduction: Large proportions of the population are not meeting recommended levels of physical activity and have increasingly sedentary lifestyles. Low levels of physical activity are predictive of poor health outcomes and time spent sedentary is related to a host of risk factors independently of physical activity levels. Building an evidence base of the best approaches to intervene in the lifestyles of inactive individuals is crucial in preventing long-term disease, disability and higher mortality rates.

Methods and analysis: Systematic searches will be conducted on all relevant databases (eg, PubMed, Scopus, CINAHL, PsycINFO). Studies will be included if they assess interventions aimed at changing physical activity or sedentary behaviour levels in adults (over 18) who are inactive and do not suffer from chronic conditions. Studies must also be randomised controlled trials (RCTs), have a primary outcome of physical activity or sedentary behaviour, and measure outcomes at least 6 months after intervention completion. Studies will be coded using the Behaviour Change Technique (BCT) taxonomy v1 and Template for Intervention Description and Replication (TIDieR) guidelines. 2 reviewers will independently screen fulltext articles and extract data on study characteristics, participants, BCTs, intervention features and outcome measures. Study quality will also be assessed independently by 2 reviewers using the Cochrane risk of bias tool. A meta-analysis will be considered if there is sufficient homogeneity across outcomes. GRADE criteria will be used to assess quality of evidence.

Dissemination: This will be the first review to systematically appraise interventions aimed at changing the physical activity or sedentary behaviour of inactive individuals using RCT designs with a 6-month followup post-intervention. This review will better inform intervention designers targeting inactive populations and inform the design of a future complex intervention. Review registration number: This protocol was registered with the International Prospective Register of Systematic Reviews (PROSPER0) on 17 October 2014 (registration number: CRD42014014321).

\section{Strengths and limitations of this study}

- This review will provide a unique contribution by being the first to evaluate the most effective behaviour change techniques (BCT) used in randomised controlled trials of interventions promoting physical activity and/or decreasing sedentary behaviour in inactive adults using the BCT taxonomy v1 and Template for Intervention Description and Replication (TIDieR) reporting guidelines.

- This review will also be the first to appraise these studies in inactive populations free of chronic conditions with a minimum of 6-month post-intervention follow-up. This will provide researchers, clinicians and the wider public with evidence of sustainable ways in which to reduce the risk factors that accompany inactive lifestyles.

- The main limitation is that there is always a possibility that the review does not identify every piece of evidence relevant to the research question, especially due to the strict inclusion criteria. Every effort will be made to search all appropriate resources to minimise this risk.

\section{BACKGROUND}

Physical inactivity is the fourth leading risk factor for mortality around the world. ${ }^{1}$ Walking, moderate and vigorous physical activity have a beneficial effect on the risk factors associated with cardiovascular disease (CVD), type 2 diabetes and cancer. $^{2}$ Participating in 150 min per week of vigorous intensity physical activity is related to better survival rates and better physical and cognitive health in older age. ${ }^{3}$ When compared with respondents who participate in low levels of activity, highly active (men, 22\%; women, 31\%) and moderately active (men, $19 \%$; women, 24\%) people have a reduced risk of all-cause mortality. ${ }^{4}$ Even relatively low 
amounts of leisure-time physical activity (92 min/week) have been associated with a $14 \%$ reduction in risk of mortality and increased life expectancy of 3 years compared with no activity. ${ }^{5}$ The evidence is clear that physical activity is highly beneficial, yet only $67 \%$ of men and $55 \%$ of women in England report participating in the recommended levels of physical activity in 2012, ${ }^{6}$ with corresponding figures of $52 \%$ and $43 \%$ from the USA in $2008 .^{7}$ Objectively measured levels in 2008 from the UK data show much lower levels-just $6 \%$ of men and $4 \%$ of women performed the recommended amount, ${ }^{6}$ demonstrating the over-reporting inherent in self-report measures of this type. ${ }^{8}$

The latest research also suggests that alongside the negative health outcomes related to low physical activity levels, sedentary behaviour is an independent risk factor for a range of health problems. Sedentary behaviour (in this case, watching television) is related to obesity even after controlling for levels of leisure-time physical activity and diet. ${ }^{9}$ Daily sitting time is linked to all-cause mortality $^{10}$ and risk of mortality through $\mathrm{CVD}^{11}$ after factoring in physical activity levels. Higher levels of sedentary behaviour are also predictive of insulin resistance ${ }^{12}$ and strongly associated with diabetes. ${ }^{13}$ Despite these unequivocal data, many people report sitting for longer than $5 \mathrm{~h}$ per day, with objective measurements suggesting that sedentary activities comprise $57 \%$ of daily behaviour in Australian adults, ${ }^{14}$ and between $52 \%$ (30-39 years old) and $67 \%$ (70-85 years old) in US adults. ${ }^{15}$ Interventions aimed at increasing physical activity and/ or reducing sedentary behaviour are clearly of paramount importance, yet are often described poorly and have shown modest results, particularly in the long term (eg, over a 12-month follow-up ${ }^{16}$ ).

There have been a wide range of systematic reviews of interventions in this area that have had a narrow focus, for instance, on only one approach such as pedometer provision, ${ }^{17}$ one mode of delivery such as telephonebased $^{18}$ or mobile technology, ${ }^{19}$ or community-based approaches only. ${ }^{20}$ Others have included nonrandomised and non-controlled designs, ${ }^{21}$ included a mixture of inactive and active populations ${ }^{22}$ or did not analyse the behaviour change techniques (BCT) that may have been related to effectiveness. ${ }^{23} 24$ Even those reviews that have included only randomised controlled trials (RCTs) have not analysed BCTs. ${ }^{25}$ When BCTs have been analysed, non-randomised designs have been included, older and less exhaustive taxonomies have been applied, and studies that did not measure outcomes for at least 6 months post-intervention have been included. $^{1626}$

Other reviews have included inactive participants as part of highly heterogeneous intervention samples including those with diabetes, CVD and depression. ${ }^{25} 23$ The BCTs that are effective with a person suffering from diabetes may be different from those used with a participant suffering from CVD or cancer. For this reason, only inactive participants who are not suffering from major or chronic diseases or conditions will be included in this review.

The biggest problem in evaluating complex interventions that attempt to change behaviour is establishing the effectiveness of various components due to the imprecise nature of the intervention content and BCT descriptions. A group of international experts have recently collaborated to produce an exhaustive taxonomy of BCTs, which names active behaviour change intervention techniques that cannot be reduced to smaller components, such as goal setting or selfmonitoring of behaviour. ${ }^{27}$ The BCT taxonomy v1 is a 93-item list which allows all behaviour change interventions to be systematically described, reviewed and replicated.

Since previous systematic reviews of physical activity and sedentary behaviour interventions have been published, ${ }^{16}{ }^{26}$ the list of BCTs that can be investigated has become broader and more nuanced. It is therefore important to find out whether the other BCTs included in this enhanced taxonomy are used and effective in physical activity and/or sedentary behaviour interventions. Another major problem in assessing the effectiveness of physical activity interventions is that details other than the BCTs have often been poorly reported. This includes vague descriptions of the rationale, materials, mode, intensity, and duration of delivery, providers or fidelity. This review will therefore also code intervention descriptions using the 'Template for Intervention Description and Replication' (TIDieR ${ }^{28}$ ). TIDieR is a 12-item checklist detailing how to report the why, what, who and where of intervention delivery. It is designed to help ensure that health professionals and researchers can accurately implement and replicate interventions and will be used alongside the BCT taxonomy v1 to evaluate included studies.

\section{Objectives}

This is the first review to systematically identify and evaluate the effectiveness of the BCTs used in RCTs of interventions aimed at increasing physical activity and/ or reducing sedentary behaviour in inactive adults with a 6-month follow-up measurement that considers all settings and modes of delivery. The aim of this systematic review is to answer the following two questions:

- What are the commonly used and most effective BCTs in RCTs of behavioural interventions to promote physical activity and/or reduce sedentary behaviour in inactive adults?

- Which intervention features are associated with intervention effectiveness, including mode of delivery, theoretical framework, dose, intensity and frequency?

\section{METHODS AND ANALYSIS}

This protocol has been reported using the Preferred Reporting Items for Systematic Reviews and MetaAnalyses Protocols (PRISMA-P) guidelines. ${ }^{29}$ 


\section{Eligibility criteria}

\section{Study characteristics}

This review will include published, unpublished and in-progress intervention studies from January 1990 to December 2014, in the English language only.

\section{Participants}

This review will include only studies with adult participants (18 or older) who are identified as inactive prior to the intervention. Inactivity may be defined differently by each study, but an upper limit will be set for inclusion at less than $150 \mathrm{~min}$ of moderate-intensity or $75 \mathrm{~min}$ of vigorous-intensity activity per week, or less than 10 000 steps per day. Those who are inactive due to serious injury or long-term physical incapacity will be excluded. Participants suffering or rehabilitating from serious/ chronic disease will be excluded. Those who have recovered from serious illness or injury will be considered in subgroup analysis if appropriate. Athlete participants and participants engaging in other health-related /fitnessrelated programmes will also be excluded from the review. Studies in which there is a mixture of participants (eg, some classified as inactive, and others obese or at risk) will only be considered if at least $70 \%$ are classified as inactive, making a clear distinction from any previous reviews that have used more heterogeneous samples. ${ }^{24}$

\section{Intervention}

Studies will be included if they evaluate an RCT intervention that aims to increase physical activity and/or reduce sedentary behaviour as one of its primary aims. Intervention approaches must include at least one BCT from the BCT taxonomy $\mathrm{v}^{27}$ but do not need to have a specified theoretical basis. Interventions can be delivered in a variety of settings (eg, community centre, hospital, clinic, private residence), modes of delivery (eg, face-to-face, online, text message, phone call) or frequency, duration or intensity (anywhere from a single contact point of $5 \mathrm{~min}$ to intensive year-long or more interventions). Excluded are studies that do not attempt to directly change physical activity and/or sedentary behaviour. For example, interventions that only measure change in intentions and not behaviour itself.

\section{Comparator or control}

This review will include studies that compare a physical activity and/or a sedentary behaviour intervention that contains at least one BCT to any of the following controls: passive control group (eg, usual care, waiting list control, no treatment) or active control group (eg, alternative cognitive or behavioural approaches).

\section{Outcome measures}

Primary outcomes: Studies will be included if one of the primary outcomes is objective or self-reported physical activity and/or sedentary behaviour assessed by standardised subjective or objective tools between baseline, post-intervention and a 6-month follow-up. This review will not include studies reporting only on changes in weight or well-being. Outcomes should be measured for a minimum of 6 months after intervention completion.

Secondary outcomes: For the studies that meet the principal inclusion criteria, the following outcomes will also be assessed if available: objectively measured health indicators (eg, body mass index, BMI), subjective well-being (life satisfaction, health-related quality of life, positive and negative affect), self-efficacy and metabolic health (eg, blood pressure, lipids, insulin resistance). Adverse effects will also be reported where available (eg, injury risk, worsening health inequalities).

\section{Information sources}

This review will include comprehensive searches on the following electronic databases: PubMed, Scopus, CINAHL, Applied Social Sciences Index (ASSIA), PsycINFO, Web of Science, SPORTDiscus, EMBASE, Cochrane Central Register of Controlled Trials (CENTRAL), British Nursing Index (BNI), Google Scholar and the Health Technology Assessment (HTA) database. All databases will be searched between January 1990 and December 2014.

In addition to the electronic database searches, we will search for published systematic reviews of physical activity interventions to identify relevant RCTs; reference lists of relevant articles and books; the Database of Abstracts of Reviews of Effects (DARE); the Cochrane systematic review database; the National Institute of Health Research (NIHR) portfolio for recently completed or ongoing studies; the current controlled trials register; and the System for Information on Grey Literature (SIGLE). Furthermore, we will hand search the bibliographies of all included studies and request from experts in the field any relevant information on unpublished and ongoing research, and key related journals.

\section{Search strategy}

Searches will include a combination of terms from medical subject headings ( $\mathrm{MeSH}$ ) and keywords in the title, abstract and text for the population, intervention, comparator and outcomes. A PubMed search is included in table 1 -this will be adapted to the syntax and subject headings of the remaining databases.

\section{Data management}

The results from all literature searches will be imported into Endnote reference management software. Duplicates will be removed by the software and then the main reviewer will manually remove any other examples.

\section{Selection process}

One reviewer will screen all retrieved records by title and abstract for all inclusion and exclusion criteria. A second reviewer will also screen a random $10 \%$ of the total titles and abstracts. Any disagreements at this stage will be included for further assessment. Following initial screening, full-text versions of all potentially relevant studies will be retrieved and reviewed independently for 


\begin{tabular}{|c|c|}
\hline Concept & Search terms \\
\hline Population & $\begin{array}{l}\text { MeSH terms: adult (exp), body weight, body mass index, sedentary lifestyle, overweight (exp) } \\
\text { Free text terms: BMI, inactive, sedentary }\end{array}$ \\
\hline Intervention & 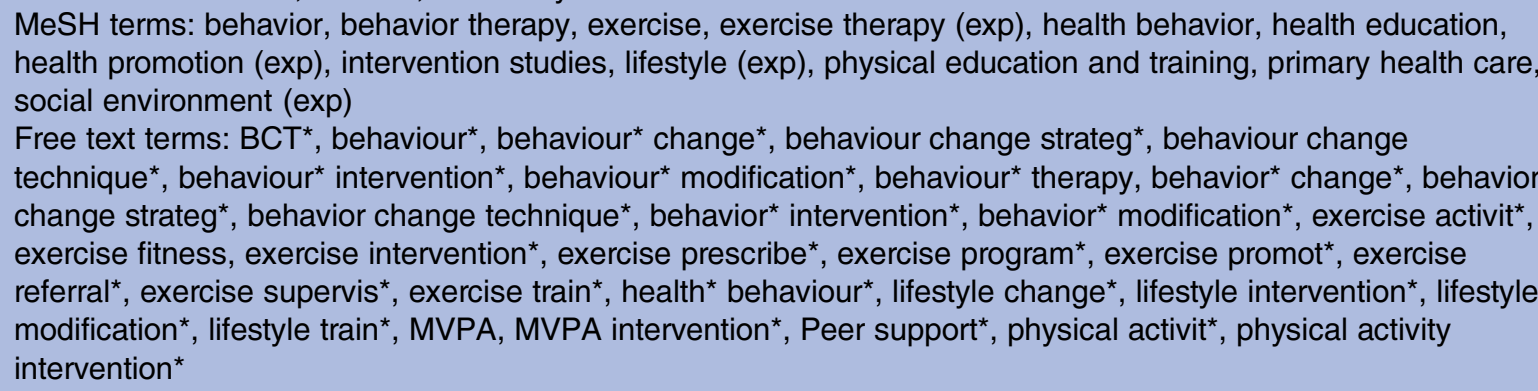 \\
\hline Comparator & $\begin{array}{l}\text { MeSH terms: clinical trials } \\
\text { Free text terms: Clinical trial [pt], placebo [ab], randomly [ab], randomized [ab], trial [ti] }\end{array}$ \\
\hline Outcomes & $\begin{array}{l}\text { MeSH terms: exercise, physical fitness, resistance training, sports, walking } \\
\text { Free text terms: MVPA, physical activit }{ }^{*} \text {, physical inactivit*, sedentary behaviour }{ }^{*}, \text { sedentary behavior }^{*} \text {, fitness }\end{array}$ \\
\hline
\end{tabular}

suitability by two reviewers. Study authors will be contacted where necessary if relevant information on eligibility is missing. Reasons for inclusion and exclusion will be recorded, and a group discussion will resolve any discrepancies following a blind review by a third author.

\section{Data extraction}

Two reviewers will independently extract data from included studies into Excel using a data extraction form. The form will be piloted on a sample of studies external to the review to ensure consistency of extraction between authors. Any disagreements will be resolved by discussion with a third author and/or by seeking further clarification from study authors.

\section{Data items}

Two reviewers will code and extract data independently using the following categories:

- General: date of data extraction, author(s), article title, type of publication, country of origin, and source of funding.

- Study characteristics: aims/objectives of the study, study design (including control groups), inclusion and exclusion criteria, recruitment and sampling methods (including unit of randomisation and blinding), and unit of allocation.

- Participants: population type and setting, inclusion and exclusion criteria, number of participants, and baseline characteristics (eg, age, gender, weight status, ethnicity, socioeconomic status and health/risk factors).

- Features of interventions: intervention setting, care provider, code BCTs based on the BCT taxonomy $\mathrm{v},{ }^{27}$ code for the TIDie ${ }^{28}$ guidelines for reporting of interventions, and theoretical basis.

- Measurement description: unit of measurement, type of measurement used (objective/subjective), additional outcomes measured (eg, mood and life satisfaction), follow-up duration, and frequency.
When possible, we will include results that have used intention-to-treat analysis and if effect sizes cannot be calculated, further information will be sought from study authors.

\section{Outcomes and prioritisation}

The primary outcomes are physical activity and sedentary behaviour. Physical activity outcomes may include objectively measured pedometer steps (per day/week), or accelerometer data. Cardiorespiratory fitness is also acceptable as an objective outcome of changes in physical activity $\left(\mathrm{eg}, \mathrm{VO}_{2} \max ^{30}\right)$. Self-report physical activity outcomes may include walking (steps per day/week), vigorous-intensity, moderate-intensity and/or lightintensity activity (minutes per day/week), and proportion of participants achieving recommended physical activity levels (eg, $150 \mathrm{~min} /$ week of moderate-intensity activity). Sedentary behaviour outcomes may include objectively measured sedentary behaviour or sitting time using accelerometers or inclinometers. Self-report sedentary behaviour outcomes may include time spent watching TV, computer usage, total screen time (TV, computer and phone/iPad use combined) or sitting (travel, relaxing and workplace). This review will not include studies reporting only on changes in weight or well-being. Outcomes should be measured for a minimum of 6 months after intervention completion.

For the studies that meet the principal inclusion criteria, the following outcomes will also be assessed if available: objectively measured BMI and metabolic health (eg, blood pressure, lipids, insulin resistance), subjective well-being using validated questionnaires such as life satisfaction (eg, SWLS ${ }^{31}$ ), health-related quality of life (eg, MOS SF- $\left.36^{32}\right)$, positive and negative affect (eg, PANAS $^{33}$ ) and self-efficacy (eg, GSES ${ }^{34}$ ).

\section{Risk of bias in individual studies}

Two reviewers will independently assess the methodological quality of the studies using the Cochrane tool for 
assessing risk of bias. ${ }^{35}$ This tool evaluates the quality of allocation sequence generation and concealment, blinding of participants, intervention providers and outcome assessors, completeness of data, the extent to which outcomes are selectively reported, and any other potential sources of bias. Each domain will be assigned a risk of bias category from the following: 'low risk for bias', 'unclear risk for bias' and 'high risk for bias'. Information on quality for each study will be accompanied by a description of the assessment and decision-making process.

\section{Data synthesis}

Differences in effectiveness will be analysed according to outcomes and the number and type of BCTs used. ${ }^{36}$ Appropriate statistical techniques will be used for each type of continuous (weighted mean differences if outcomes are consistent or standard mean difference if different outcomes are used, with 95\% CI) and dichotomous variables (risk ratios, with 95\% CI). This review will also include a meta-analysis (if there is sufficient homogeneity of outcomes) to calculate pooled effect sizes across studies, using a random-effect or fixed-effect model depending on the level of heterogeneity of intervention effects. Heterogeneity will be investigated using $\chi^{2}$ (significance level: 0.1 ) and Higgins $\mathrm{I}^{2}$ statistics, with high levels (as specified by guidance in the Cochrane Handbook for Systematic Reviews of Interventions) being considered suitable for subgroup analysis to determine the source of the heterogeneity.

\section{Subgroups and sensitivity analysis}

Analysis by subgroups will include (if possible or appropriate) the following: mode of delivery (eg, face-to-face or internet-delivered); type of physical activity/sedentary behaviour measurement (self-report vs objective); BCTs; theoretical basis; targeting single versus multiple health behaviours; age of participants (over 65 vs under 65 years). Sensitivity analysis will be carried out to determine the effects of studies with a high risk of bias on the overall results with and without these studies.

If a meta-analysis is not possible, a narrative synthesis of all relevant studies will be conducted, including tables of study characteristics, participant and intervention details, settings and outcomes.

\section{Meta-bias}

This review will assess study protocols for outcome reporting bias by judging whether authors have selectively reported outcomes using the Cochrane tool for assessing risk of bias. ${ }^{35}$ Reporting bias will be analysed using funnel plots.

\section{Confidence in cumulative evidence}

The quality of evidence for primary outcomes will be assessed using the Grading of Recommendations Assessment, Development and Evaluation guidelines, ${ }^{37}$ which include the following domains: design; study limitations; consistency; directness; precision and publication bias. Quality will be judged as high (we are very confident that the true effect lies close to that of the estimate of the effect), moderate (we are moderately confident in the effect estimate: the true effect is likely to be close to the estimate of the effect, but there is a possibility that it is substantially different), low (our confidence in the effect estimate is limited: the true effect may be substantially different from the estimate of the effect), or very low (we have very little confidence in the effect estimate: the true effect is likely to be substantially different from the estimate of effect). ${ }^{38}$

\section{DISCUSSION}

Inactive populations are a key intervention target as they are at risk of a host of negative health outcomes, even when controlling for physical activity levels. To date, no review of physical activity or sedentary behaviour interventions has focused exclusively on inactive populations, RCTs or coded BCTs exhaustively using the most recent BCT taxonomy v1. This review will be the first to evaluate the effectiveness of BCTs used in RCTs of physical activity and/or sedentary behaviour interventions in populations of inactive adults using the latest and most comprehensive taxonomies and reporting guidelines with a 6-month follow-up. It is highly important to use the latest coding guidelines to assess the effectiveness of previous interventions, and to inform future interventions for people with inactive lifestyles before they develop chronic conditions that place such a large burden on individuals and society in terms of personal, social and economic costs. This review also represents preliminary work for the development and evaluation of a future complex intervention, consistent with the guidelines from the Medical Research Council. ${ }^{39}$

Contributors All authors contributed to the development of the selection criteria. NH developed the search strategy. AC provided expertise on the physical activity literature, BCT taxonomy and TIDieR framework considerations. DT provided expertise on systematic review methodology and the risk of bias assessment. NT contributed to the data extraction criteria. All authors read and provided feedback on the draft and approved the final manuscript.

\section{Competing interests None declared.}

Provenance and peer review Not commissioned; externally peer reviewed.

Data sharing statement This systematic review protocol was registered with the International Prospective Register of Systematic Reviews (PROSPERO) on 17 October 2014 (registration number: CRD42014014321). This protocol has been reported using the Preferred Reporting Items for Systematic Reviews and Meta-Analyses Protocols (PRISMA-P) guidelines. The final review and data will be published later.

Open Access This is an Open Access article distributed in accordance with the Creative Commons Attribution Non Commercial (CC BY-NC 4.0) license, which permits others to distribute, remix, adapt, build upon this work noncommercially, and license their derivative works on different terms, provided the original work is properly cited and the use is non-commercial. See: http:// creativecommons.org/licenses/by-nc/4.0/ 


\section{REFERENCES}

1. World Health Organization. Global recommendations on physical activity for health. Geneva, Switzerland: WHO Press, 2010.

2. Baumann AE. Updating the evidence that physical activity is good for health: an epidemiological review 2000-2003. J Sci Med Sport 2004;7:6-19.

3. Almeida OP, Khan KM, Hankey GJ, et al. 150 minutes of vigorous physical activity per week predicts survival and successful ageing: a population-based 11-year longitudinal study of 12201 older Australian men. Br J Sports Med 2014;48:220-5.

4. Löllgen $H$, Böckenhoff $A$, Knapp $G$. Physical activity and all-cause mortality: an updated meta-analysis and different intensity categories. Int J Sports Med 2009;30:213-24.

5. Wen CP, Wai JPM, Tsai MK, et al. Minimum amount of physical activity for reduced mortality and extended life expectancy: a prospective cohort study. Lancet 2011;378:1244-53.

6. Health and Social Care Information Centre. Statistics on obesity, physical activity and diet. England, 2014.

7. Centre for Disease Control and Prevention. http://www.cdc.gov/ physicalactivity/data/facts.html (accessed 13 Apr 2015).

8. Lee PH, Macfarlane DJ, Lam TH, et al. Validity of the international physical activity questionnaire short form (IPAQ-SF): a systematic review. Int J Behav Nutr Phys Act 2011;8:115

9. Shields M, Tremblay MS. Sedentary behaviour and obesity. Health Rep 2008;19:19-30.

10. Chau JY, Grunseit AC, Chey T, et al. Daily sitting time and all-cause mortality: a meta-analysis. PLOS ONE 2013;8:e80000.

11. Katzmarzyk PT, Church TS, Craig CL, et al. Sitting time and mortality from all causes, cardiovascular disease, and cancer. Med Sci Sports Exerc 2009;41:998-1005.

12. Helmerhorst $\mathrm{HJ}$, Wijndaele K, Brage S, et al. Objectively measured sedentary time may predict insulin resistance independent of moderateand vigorous-intensity physical activity. Diabetes 2009;58:1776-9.

13. Wilmot EG, Edwardson CL, Achana FA, et al. Sedentary time in adults and the association with diabetes, cardiovascular disease and death: systematic review and meta-analysis. Diabetologia 2012;55:2895-905

14. Healy GN, Wijndaele K, Dunstan DW, et al. Objectively measured sedentary time, physical activity, and metabolic risk. Diabetes Care 2008;31:369-71.

15. Matthews CE, Chen KY, Freedson PS, et al. Amount of time spent in sedentary behaviours in the United States, 2003-2004. Am J Epidemiol 2008;167:875-81.

16. Greaves C, Sheppard KE, Abraham C, et al. Systematic review of reviews of intervention components associated with increased effectiveness in dietary and physical activity interventions. BMC Public Health 2011:11:119.

17. Brevata DM, Smith-Spangler C, Sundaram V, et al. Using pedometers to increase physical activity and improve health: a systematic review. JAMA 2007;298:2296-304.

18. Eakin EG, Lawler SP, Vandelanotte C, et al. Telephone interventions of physical activity and dietary behaviour change. Am J Prev Med 2007;32:419-34.

19. Free C, Phillips G, Galli L, et al. The effectiveness of mobile-health technology-based health behaviour change or disease managemen interventions for health care consumers: a systematic review. PLoS Med 2013;10:e1001362.

20. Baker PRA, Francis DP, Soares J, et al. Community wide interventions for increasing physical activity. Cochrane Database Syst Rev 2011;(13):CD008366.

21. Wilcox S, Parra-Medina D, Thompson-Robinson M, et al. Nutrition and physical activity interventions to reduce cardiovascular disease risk in health care settings: a quantitative review with a focus on women. Nutr Rev 2001;59:197-214.

22. Martin A, Fitzsimons C, Jepson R, et al. Interventions to influence sedentary behaviour in adults: systematic review and meta-analysis. Br J Sports Med 2015. Published Online First: 23 Apr 2015. doi:10.1136/bjsports2014-094524

23. Pavey TG, Taylor AH, Fox KR, et al. Effect of exercise referral schemes in primary care on physical activity and improving health outcomes: systematic review and meta-analysis. BMJ 2011; 343:d6462.

24. Prince SA, Saunders TJ, Gresty K, et al. A comparison of the effectiveness of physical activity and sedentary behaviour interventions in reducing sedentary time in adults: a systematic review and meta-analysis of controlled trials. Obes Rev 2014;15:905-19.

25. Orrow G, Kinmonth AL, Sanderson S, et al. Effectiveness of physical activity promotion based in primary care: systematic review and meta-analysis of randomised controlled trials. BMJ 2012;344: e1389.

26. Olander EK, Fletcher $\mathrm{H}$, Williams $\mathrm{S}$, et al. What are the most effective techniques in changing obese individuals' physical activity self-efficacy and behaviour: a systematic review and meta-analysis. Int J Behav Nutr Phys Act 2013;10:29.

27. Michie S, Richardson M, Johnston M, et al. The behavior change technique taxonomy (v1) of 93 hierarchically clustered techniques: building an international consensus for the reporting of behavior change interventions. Ann Behav Med 2013;46:81-95.

28. Hoffman TC, Glasziou PP, Boutron I, et al. Better reporting of interventions: template for intervention description and replication (TIDieR) checklist and guide. BMJ 2014;348:g1687.

29. Shamseer L, Moher D, Clarke M, et al. Preferred reporting items for systematic reviews and meta-analyses protocols (PRISMA-P) 2015: elaboration and explanation. BMJ 2014;349:g7647.

30. Sloth $\mathrm{M}$, Sloth $\mathrm{D}$, Overgaard $\mathrm{K}$, et al. Effects of sprint interval training on VO2max and aerobic exercise performance: a systematic review and meta-analysis. Scand J Med Sci Sports 2013;23:e341-52.

31. Diener E, Emmons RA, Larsen RJ, et al. The satisfaction with life scale. J Pers Assess 1985;49:71-5.

32. Ware JE, Sherbourne CD. The MOS 36-item short form health survey (SF-36). A conceptual framework and item selection. Med Care 1992;30:473-83.

33. Watson D, Clark LA, Tellegen A. Development and validation of brie measures of positive and negative affect: the PANAS scales. J Pers Soc Psychol 1988;54:1063-70.

34. Schwarzer R, Jerusalem M. Generalized self-efficacy scale. In: Weinman J, Wright S, Johnston M, eds. Measures in health psychology: a user's portfolio. Causal and control beliefs. Windsor, United Kingdom: NFER-NELSON, 1995:35-7.

35. Higgins JPT, Altman DG, Gøtzsche PC, et al. The Cochrane Collaboration's tool for assessing risk of bias in randomised trials. BMJ 2011;343:d5928.

36. Martin J, Chater A, Lorencatto F. Effective behaviour change techniques in the prevention and management of childhood obesity. Int J Obes 2013;37:1287-94

37. Guyatt G, Oxman AD, Akl EA, et al. GRADE guidelines: 1. Introduction-GRADE evidence profiles and summary of findings tables. J Clin Epidemiol 2011;64:383-94.

38. Balshem $\mathrm{H}$, Helfand $\mathrm{M}$, Schunemann $\mathrm{HJ}$, et al. GRADE guidelines: 3. Rating the quality of evidence. J Clin Epidemiol 2011;64:401-6.

39. Craig P, Dieppe P, Macintyre S, et al. Developing and evaluating complex interventions: the new Medical Research Council guidance. BMJ 2008;337:a1655 